\title{
Fórmulas de tratamiento nominal en la Universidad de Antioquia. Aproximaciones al habla de estudiantes de las Facultades de Educación e Ingeniería*
}

\author{
FRANKLIN YESSID ARIAS BEDOYA** \\ franklinyessid@hotmail.com \\ MARÍA ALEJANDRA GARCÍA ROMERO ${ }^{* * *}$ \\ poe.literatura@gmail.com \\ ANA CAROLINA RUIZ VILLADA*** \\ caroruizzz@hotmail.com
}

Recepción: 23 de enero de 2015

Aprobación: 20 de abril de 2015

Forma de citar este artículo: Arias Bedoya, F. Y., García Romero, M. A., \& Ruiz Villada, A. C. (2016). Fórmulas de tratamiento nominal en la Universidad de Antioquia. Aproximaciones al habla de estudiantes de las Facultades de Educación e Ingeniería. Cuadernos de Lingüística Hispánica, (27), 57-77.

* $\quad$ Este artículo presenta resultados de la investigación intitulada Fórmulas de tratamiento nominal en estudiantes de las Facultades de Educación e Ingeniería de la Universidad de Antioquia, Colombia; proyecto orientado por el doctor en Linguiística Juan David Martínez y revisado por el doctor Robinson Grajales Alzate, director del Grupo de Estudios Sociolingüísticos (GES) de la Universidad de Antioquia, Colombia.

** Licenciatura en Educación Básica con Énfasis en Humanidades, Lengua Castellana, Universidad de Antioquia, Colombia.

**** Licenciatura en Educación Básica con Énfasis en Humanidades, Lengua Castellana, Universidad de Antioquia, Colombia

****** Licenciatura en Educación Básica con Énfasis en Humanidades, Lengua Castellana, Universidad de Antioquia, Colombia 


\section{Resumen}

En este artículo se parangonan dos comunidades discursivas pertenecientes a la Universidad de Antioquia: la Facultad de Educación y la Facultad de Ingeniería. Para ello, se emplea una metodología cualitativa sustentada en encuestas que permite detallar rasgos morfológicos y pragmasemánticos observados en las Fórmulas de Tratamiento Nominal ${ }^{1}$ utilizadas por los hablantes. Se hace hincapié en las familias léxicas y la modalidad axiológica para el contraste y análisis de la información, esto, a la luz de los planteamientos propuestos por Mervyn Lang, Manuel Casado y Ramón Almena, en lo que compete a la formación de palabras en el español y Norma Carricaburo en lo relativo a las FT'N. Se hallan entonces, dentro de los resultados, fenómenos de acortamiento, derivación, diptongación, sufijación apreciativa, inconexiones modalidad-significado, entre otros.

Palabras clave: Fórmulas de tratamiento nominal, familia léxica, campo semántico, modalidad axiológica, metaplasmos.

\section{Nominal address formulas at Universidad de Antíoquia. An aproach to the speech of students of the Faculties of Education and Engineering}

\section{Abstract}

In this article, two speech comunities from the Universidad de Antioquia are compared: the Faculty of Education and the Faculty of Engineering. A qualitative metodology supported by surveys is used for this purpose. This metodology allows for a detailed analysis of the morfological, pragmatic and semantic features observed in the nominal address formulas (NFA) used by the speakers. In order to analize and compare the information, an emphasis in the lexical families and axiological modalities is made. This method is supported by approaches to the construction of Spanish words proposed by Mervyn Lang, Manuel Casado and Ramón Almena, and also by the studies related to NFA made by Norma Carricaburo. The following phenomena may be found as results of this study: shortening, derivation, diphthongation, appreciative suffixation, and modality/meaning incoherence, among others.

Key words: nominal address formulas, lexical family, semantic field, axiological modality, metaplasms.

1 De ahora en adelante FT'N. 


\section{Formules de traitement nominal dans l'Université d'Antioquia. Approximations au parler d'étudiants des Facultés d'Éducation et de Génie}

\section{Résumé}

Dans cet article, on parangonne deux communautés discursives appartenant à l'Université d'Antioquia: la Faculté d'Éducation et la Faculté de Génie. Pour cela, on utilise une méthodologie qualitative soutenue sur des enquêtes qui permettent de détailler des traits morphologiques et pragma-sémantiques observés dans les Formules de Traitement Nominal, utilisés par les parlants. On met l'accent sur les familles lexiques et la modalité axiologique pour le contraste et l'analyse de l'infirmation. Ceci, à la lumière des énoncés proposés par Mervyn Lang, Manuel Casado et Ramón Almena, en ce qui concerne la formation de mots en espagnol, et Norma Carricaburo, par rapport aux FTN. Alors on trouve, dans les résultats, des phénomènes de: raccourcissement, dérivation diphtongaison, suffixation appréciative, déconnexions modalité-sens, entre autres.

Mots clés: formules de traitement nominal, famille lexique, champ sémantique, modalité axiologique, métaplasme.

\section{Fórmulas de tratamento nominal na Universidad de Antioquia. Aproximações à fala de estudantes das Faculdades de Educação e Engenharia}

\section{Resumo}

Neste artigo se parangonam duas comunidades discursivas pertencentes à Universidad de Antioquia: a Faculdade de Educação e a Faculdade de Engenharia. Para isso, se emprega uma metodologia qualitativa sustentada em pesquisas que permite detalhar características morfológicas e pragmasemânticas observadas nas Fórmulas de Tratamento Nominal utilizadas pelos falantes. É dado ênfase nas famílias léxicas e na modalidade axiológica para o contraste e a análise da informação, tudo isto, é realizado à luz das abordagens propostas por Mervyn Lang, Manuel Casado e Ramón Almena, no que compete à formação de palavras no espanhol e Norma Carricaburo no relativo às FT̃N. Dentro dos resultados são encontrados fenômenos de redução, derivação, ditongação, sufixação apreciativa, inconexões modalidade-significado, entre outros.

Palavras chave: fórmulas de tratamento nominal, família léxica, campo semântico, modalidade axiológica, metaplasmos. 


\section{Introducción}

La sociolingüística, entendida como "área de la linguística que se ocupa de las relaciones entre lenguaje y la sociedad, y de los estudios hechos de los códigos en su contexto social (más que en despachos y laboratorios)" (Trudgill, 1975, p. 28), se encarga de estudiar desde la investigación empírica gestas de la lengua (morfológicas, sintácticas, semánticas, lexicales, fonéticas...) en una comunidad de habla determinada, esto es, busca "examinar las correlaciones entre variables lingüísticas y variables sociales, analizar las variables que sufre una lengua en relación con los usuarios y los contextos sociales de su uso" (Moreno, 1998, p. 30).

Así, se centra en la variación lingüística, es decir, el conjunto de cambios y posibles realizaciones (Variantes) de un mismo elemento linguístico (Variable), el cual responde a cuestiones espaciales, cognitivas, cognoscitivas, situacionales, entre otras. Dicho de otro modo, "es la alternancia de dos o más expresiones de un mismo elemento, cuando esta no supone ningún tipo de alteración o cambio de naturaleza semántica y cuando se ve condicionada por factores lingüísticos y sociales" (Moreno, 1998, p. 33). Estos factores configuran un maremágnum de variaciones tipológicamente separadas pero conectadas de manera inexorable, distribuidas en lo diatópico, diafásico, diastrático, sincrónico y diacrónico.

En esta línea, es menester mencionar que, así como influyen elementos como el nivel de instrucción, el campo de estudio tiene incidencia directa o indirecta sobre las fórmulas comunicacionales que emplean sus integrantes. De esta manera, en el contraste de dos comunidades discursivas (CD) disímiles se pueden hallar diferencias lingüísticas tanto de orden lexical (morfológico, semántico) en la creación de palabras y la modificación de significados, como en los niveles fonético, sintáctico y pragmático. Se entiende por comunidad discursiva "un insieme di persone, di estensione indeterminata, che condividano l'accesso a un insieme di varietà di lingua (il repertorio linguistico) e che siano unite da una qualche forma di aggregazione socio-politica. L'insieme di varietà di lingua e l'estensione dell'aggregazione possono essere stabiliti di volta in volta" (Berruto 2003, p. 60). Bajo esta premisa, es relevante decir que en una indagación sociolingǘstica además de la variable "nivel de instrucción" es menester poner la mirada sobre el área disciplinar a la que se adscribe la población, pues, si bien se encontrarán elementos 
análogos entre las muestras, este componente marca ciertas diferenciaciones conceptuales y lexicográficas concretadas en la situación comunicativa. Ahora, teniendo en cuenta lo enunciado, la investigación exploratoria de la que se desprende este artículo compara dos comunidades discursivas pertenecientes a la Universidad de Antioquia: por un lado, la Facultad de Educación, y por el otro, la Facultad de Ingeniería, esto, con el fin de observar ciertas Fórmulas de Tratamiento Nominal y su monto de producción en ambos contextos.

El estudio de las FT'N en el habla de Medellín ha sido explorado con anterioridad por el investigador Milton Daniel Castellano. A continuación se enumeran algunos de sus trabajos, pues, se instituyen como antecedentes clave en el estudio del tema en esta población:

- "Fórmulas de tratamiento nominales para la pareja en el habla juvenil medellinense" (pp. 166 - 181). En este texto se exploran los apelativos usados en parejas cuyas edades oscilan entre los 17 y los 25 años, así como los elementos extralingüísticos (contextuales) que influyen en la interpretación de sus FTN.

- "Caracterización morfológica de las fórmulas de tratamiento nominales en el habla de Medellín" (pp. 221 - 346). Artículo que toma como eje el análisis de los procesos morfológicos para la producción de FTN a partir de una perspectiva lexicográfica.

- “Cortesía verbal y fórmulas de tratamiento nominales: Análisis pragmático de las fórmulas de tratamiento nominales en el habla de Medellín (pp. 41 - 56). Se realiza una caracterización linguística de las FTN y un análisis pragmático de las relaciones entre dicho término y la cortesía verbal. Allí se resalta la ambivalencia de los apelativos dentro del continuum linguístico, ya que, formas nominales similares son usadas tanto en la cortesía negativa como positiva, e incluso, en la descortesía.

Vale decir que, estos trabajos se centran en el habla coloquial de muestras genéricas aleatorias, donde, si bien algunos consideran cuestiones diastráticas como edad y género, dejan de lado, por reducción metodológica, el nivel y campo de instrucción de los hablantes. Otras investigaciones sobre el tema, estas a nivel internacional, como "Un estudio sociolingüístico. Sistemas de tratamiento de la juventud de Valladolid" de Juan Manuel Pedroviejo Esteruelas; "Tratamiento y juventud de la lengua hablada. Aspectos sociolinguísticos" de Diego de Alba y Sánchez Lobato; y "Notas comparativas sobre el tratamiento en español y rumano" de Dumitrscu, revelan datos que coinciden con los análisis realizados por Castellanos y los resultados de nuestra investigación. 


\section{Marco metodológico}

Dado que, lo que se buscó en la investigación fue la descripción de la variación léxica que se presenta en las Fórmulas de Tratamiento Nominal (FTN) de dos espacios académicos específicos, sin un énfasis en la representatividad numérica, se siguió una metodología cualitativa-exploratoria. Como punto de partida, se entiende por Fórmulas de tratamiento (FT) a "todas aquellas estructuras basadas en la combinación de elementos léxicos y gramaticales que los hablantes de una determinada lengua utilizan para apelar a sus semejantes" (Molina, 2002, p. 97). Entre estos aspectos destacan las formas pronominales: tú, usted y vos; y las nominales, conformadas por aquellas realizaciones lingüísticas constituidas por sintagmas nominativos, sustantivos $\mathrm{u}$ otras categorías sustantivadas que poseen un sentido apelativo. Para Carricaburo (1997, p. 49) las FTN pueden ser de tipo denotativo, o connotativo: el primer concepto alude llanamente al nombre y su correspondencia directa con el sujeto, mientras que el segundo se nutre de las distintas marcas, relaciones, rasgos y signos que se le atribuyen al destinatario. Estos componentes dilucidan, por lo tanto, vínculos y atributos de familiaridad, afectividad, lejanía, edad, inteligencia, fisionomía, procedencia étnica, entre otros.

Atendiendo al propósito explicitado anteriormente, se plantean a continuación algunas consideraciones metodológicas bajo las cuales se ha realizado la investigación. Posteriormente, se exponen de manera teórico-analítica los resultados más significativos hallados en la pesquisa: desde una lógica del significado y la modalidad axiológica (valores y campos semánticos) y desde la óptica morfológica en lo referente a los metaplasmos y las familias léxicas. Finalmente, se presentan algunas conclusiones e interrogantes al respecto.

Un primer momento de esta, consistió en la selección del lugar de estudio, la elección de los participantes, la definición de la fuente (escrita), y el diseño del instrumento de investigación. Este último, en formato cuestionario ostentó dos fines cardinales: la recolección sistemática de las diferentes Formas de Tratamiento Nominal, por una parte y, la recopilación categórica de las mismas de acuerdo con un sistema modal axiológico determinado, por otra.

El segundo momento de la investigación está directamente relacionado con los criterios aplicados en la delimitación de los informantes, así como en el establecimiento de la estructura general de la encuesta, donde, desde la perspectiva de las variaciones lingüísticas, destacan lo lexical, morfológico y semántico-pragmático, mientras que desde la óptica extralinguística se acentúan la variación diastrática, eligiéndose jóvenes entre los 18 y 25 años de edad que cursan los 3 primeros semestres de su carrera y que oscilan entre los estratos socioeconómicos 1 y 3 ; y diafásica, pues, se aluden a distintas situaciones 
comunicativas para la construcción y recolección de los conceptos propios de los hablantes en escenarios como papelerías, filas, noviazgos, amistades, etc.

El tercer momento de la metodología radicó en la recolección y clasificación de la información, la cual se organizó en una rejilla de Excel diferenciando Facultades y géneros, donde se consignaron las FTN teniendo en cuenta su número de repeticiones, usos y variantes entre la población muestra. Posteriormente, se procedió con la clasificación de las muestras atendiendo a las dos líneas que se han elegido para el análisis de las mismas: léxico-morfológico y semántico-pragmático. Dado que es relevante observar las múltiples realizaciones que se producen de las palabras, en la primer linde se ordenaron los hallazgos por familias léxicas, sistematizándose así: (1) el conjunto de palabras que no podían incluirse en ninguna familia léxica, evidenciando de estas el lexema, uso por número de persona (U*NP) y porcentaje dentro de la población (omitido en este artículo por la limitación espacial); (2) El grupo de vocablos que presentaron fenómenos morfológicos específicos; (3) Otras categorías: apodos, nombres propios y acortamiento de los nombres. La descomposición morfológica se realiza con base en las categorías y la terminología propuesta por Mervyn Lang (1992), Manuel Casado (1999) y Ramón Almena (1999). Para la segunda línea de sentido, semántico-pragmático, que comprende la categorización y análisis de las palabras en función de su axiología: valor afectivo con carácter peyorativo, afectivo con carácter positivo, y de lejanía entre conocidos y desconocidos; se hallan los diferentes campo semánticos desde donde provienen las FTN resemantizadas para observar la preponderancia de ciertas "cadenas léxicas" en los apelativos empleados por la población muestra.

Finalmente, y como último momento, se procedió al contraste de los datos entre facultades y géneros, formulándose algunas conclusiones e interrogantes para futuras investigaciones.

\section{Modalidad axiológica y campos semánticos de origen}

Se define modalidad axiológica como la actitud expresada por el hablante respecto del enunciado que profiere, la cual corresponde a un sistema de valores específico utilizado para realizar apreciaciones tácitas o explícitas de la realidad.

Para efectos del presente análisis, el valor, entendido como núcleo de significados atravesados por intenciones determinadas que dejan entrever intimidad, distancia social y relaciones particulares entre los individuos, adquiere importancia, pues, a partir de diversas categorías se involucran tanto la significación social que tiene el vocablo, como los actos de habla que expresan estimaciones de la otredad bajo diferentes relaciones. De esta manera y, siguiendo a Castellano (2013, p. 22), la idea que autores como Arcelus (1978), 
Flores (1954) y Fontanella (1999) sostienen, acerca de la carencia semántica propia de las FTN, pierde validez en cuanto, como se verá a continuación, dichas formulaciones están relacionadas "con una combinación de procesos semánticos tales como la ampliación léxica que el hablante produce con la intención de generar ciertos efectos en el oyente, efectos que en cierta medida atienden a estrategias del uso lingüístico, es decir, al plano pragmático" (Castellano, 2013, p. 22).

\subsection{Valor afectivo con carácter peyorativo:}

Se debe tener en cuenta que los tratamientos amistosos en los que existe un alto grado de cercanía entre los hablantes, ostentan notables variaciones en lo que al valor se refiere, ya que, pueden pasar de categorías que en la norma social son consideradas como despectivas a otras que poseen un carácter positivo sobre el receptor. Así, y para el primer caso, se encuentran FTN diversas que muestran la naturaleza axiológica de las palabras:

\begin{tabular}{|c|c|c|c|c|c|c|c|}
\hline \multicolumn{4}{|c|}{ Facultad de Educación (FE) } & \multicolumn{4}{|c|}{ Facultad de Ingeniería (FI) } \\
\hline \multicolumn{2}{|c|}{ Hombres (H) } & \multicolumn{2}{|c|}{ Mujeres (M) } & \multicolumn{2}{|c|}{ Hombres (H) } & \multicolumn{2}{|c|}{ Mujeres (M) } \\
\hline$F T N$ & $U^{*} N P$ & $F T N$ & $U^{*} N P$ & FTN & $U^{*} N P$ & $F T N$ & $U^{*} N P$ \\
\hline Gonorrea & 3 & Desgraciada & 2 & Carechimba & 5 & Estúpido & 2 \\
\hline Güevón & 7 & Estúpida & 3 & Caremondá & 3 & Güevón & 2 \\
\hline Malparido & 2 & Grilla & 2 & Gonorrea & 9 & Idiota & 2 \\
\hline Marica & 7 & Güevón & 3 & Güevón & 11 & Maldito & 2 \\
\hline Perra & 2 & Malditas & 2 & Hijueputa & 6 & Malparido & 2 \\
\hline Pirobo & 2 & Malparida & 2 & Loco & 2 & Marica & 6 \\
\hline Vacío & 4 & Perra & 2 & Malparido & 4 & Pendejo & 3 \\
\hline \multirow[t]{7}{*}{ Otras } & $\ldots$ & Tonto (a) & 2 & Marica & 11 & Perris & 2 \\
\hline & & Vacío & 6 & Maricón & 3 & Porquería & 2 \\
\hline & & Zorra & 3 & Marranomono & 2 & Vacío & 3 \\
\hline & & Otras & & Perra & 2 & Otras & $\ldots$ \\
\hline & & & & Perro & 2 & & \\
\hline & & & & Pirobo (a) & 4 & & \\
\hline & & & & Otras & $\ldots$ & & \\
\hline Total: 26 & 46 & Total: 26 & 44 & Total: 37 & 91 & Total: $\mathbf{2 3}$ & 38 \\
\hline
\end{tabular}

Tabla 1: FTN afectivo-peyorativas

Nótese que, en este asunto, respecto de la relación significado, significado pragmático y valor, propio de cada palabra, puede existir una "inconexión" entre (1) el ser literal de la expresión (semántica), (2) el sentido que adquiere en contexto (pragmática) y (3) la reconfiguración en el espacio de actualización; lo cual lleva a pensar, en muchos casos, por ejemplo, no solo en la voz perra como un sustantivo femenino que alude a un animal, sino también, en el segundo caso, como un apelativo para una persona promiscua, y en tercero, a una forma con valor afectivo en la que se atenúan ambos significados. 
Mencionado lo anterior, es necesario decir que las palabras del cuadro número uno provienen de campos semánticos (CS) numerosos, los cuales han adquirido un matiz despreciativo en la norma social, pero que, aun así, se utilizan para fines no completamente displicentes. Vale decir que, hablar de este tipo de campo remite directamente a la clasificación de los vocablos según la cadena léxica de la que se producen, indiferente del uso particular que se les está dando en las comunidades muestra.

Antes que nada, si se pone la mirada sobre la Facultad de Educación, el inventario completo de la investigación deja entrever que categorías como Sexualidad: (H) chimbo, gay, güeva, güevón, marica, maricón. (M) Güevón, marica, mariconcita; Animalidad: (H) zorra, perra. (M) Burro, grilla, grillis, perro, perra, zorra; Prostitución: (H) hijueputa, zunga, ramero, puto. (M) malparida; Inteligencia o razón: (H) idiota, pendejo. (M) Boba, bruta, estúpido, idiota, imbécil, pendeja, tarado, tonto; Enfermedad: (H) nea, gonorrea. (M) Nea; Maldición o perversión: (H) lacra (M) Maldita Bith, Maldita buena, Malditas, desgraciadas, son los paradigmas constituyentes de este valor en dicha comunidad discursiva.

Al realizar el contraste entre la población masculina y femenina de la muestra, se encuentra que: (1) en la primera predomina el uso de FTN provenientes del CS sexual (23.91\%), mientras que en la segunda hay primacía de variantes adscritas a la valoración de la agudeza o raciocinio mental de los individuos (27.27\%). (2) El género masculino escasea de apelativos que expresan corrupción, depravación o condena $(2.17 \%)$, por su parte, el femenino carece de CS que aludan a enfermedades (2.27\%) o a prostitución (2.27\%). En cuanto al valor que tienen dichas palabras peyorativas en la norma de Medellín, se observa que (3) los varones hacen uso de terminologías con mayor grado de obscenidad que las féminas. (4) El número de variantes es el mismo (en la tabla completa) y la usanza de estas difiere en 2 . Finalmente, se encuentra mayor abstinencia para la enunciación de dichas FTN por parte de las jóvenes (13.64\%) que de los estudiantes hombres (8.70\%).

En cuanto a la Facultad de ingeniería, se localizan entre los campos semánticos de origen tópicos similares a los anteriores, con diferenciaciones en sus componentes y cantidades. Se tienen Sexualidad: (H) caremondá (cara de pene), soplamondá, chimbón (Cualidad de tener el falo grande), chupavergas, carechimba (cara de vagina), gay, güeva, güevón, marica, maricón, travis (travesti). (M) Güeva, güevón, marica; Animalidad: (H) zorra, mico, marranomono, marranopatrón, perra, perris, perro, abortomula. (M) chinche, perra, perris; Prostitución: (H) hijueputa. (M) Zunga; Inteligencia o razón: (H) brutus. (M) Estúpido, idiota, pendejo, sonso; Enfermedad: (H) nea, gonorrea; Comida: (H) careñame, cochovis (Bizcocho). (M) Chinchurria; Compañerismo: (H) ñero, parce, parcero. (M) Gransosioso (Gran socio), parce; y una variante proveniente del parlache $(\mathrm{H})$ ñiompiro. 
Paragonando ambos géneros se encuentra que en los hombres predomina el uso de FTN provenientes de la red léxica sexual (42.85\%) y de la correspondiente a los animales (12.08\%), mientras que en las mujeres se nota preponderancia de variantes adscritas a la "valoración" de la agudeza o raciocinio mental de los individuos (18.42\%). En el uso, existe una diferencia notable de 91 y 38 en hombres y mujeres respectivamente.

Finalmente, en este valor existen varios CS compartidos por ambas comunidades discursivas; empero, la FI posee mayor cantidad de estos, diferenciándose por tener una categoría relacionada con la comida y otra con el compañerismo. De otra parte, los hombres encuestados pertenecientes a la Facultad de Educación escasean de variantes anexas a la categoría de los animales ( 2 palabras), mientras que en la segunda comunidad se encuentran 8 realizaciones distintas de esta misma naturaleza.

Es de destacar que, el encuentro lingüístico en la Facultad de Ingeniería, donde convergen comunidades de habla distintas (mayoritariamente referentes a las costas y a Medellín), produce una mayor variación en cuanto a las FTN. Palabras como caremondá, careñame, entre otras, según el rastreo, son propias de la primera cultura, mientras que carechimba y parce son constituyentes de la segunda.

\subsection{Valor afectivo con carácter positivo:}

\begin{tabular}{|l|c|l|c|l|c|l|c|}
\hline \multicolumn{3}{|c|}{ Facultad de Educación (FE) } & \multicolumn{3}{c|}{ Facultad de Ingeniería (FI) } \\
\hline \multicolumn{2}{|c|}{ Hombres (H) } & \multicolumn{2}{c|}{ Mujeres (M) } & \multicolumn{2}{c|}{ Hombres (H) } & \multicolumn{2}{c|}{ Mujeres (M) } \\
\hline FTN & $U^{*} N P$ & \multicolumn{1}{|c|}{$F T N$} & $U^{*} N P$ & $F T N$ & $U^{* *} N P$ & $F T N$ & $U^{*} N P$ \\
\hline Acortamiento & 2 & Acortamiento & 2 & Amigo & 2 & Amor & 7 \\
\hline Amor & 6 & Amiguis & 3 & Amor & 4 & Apodo & 5 \\
\hline Apodo & 3 & Amor & 6 & Bebé & 3 & Bebé & 2 \\
\hline Corazón & 3 & Baby & 2 & Cariño & 2 & Diminutivos & 3 \\
\hline Hermano & 2 & Cariño & 3 & Corazón & 3 & Mi vida & 2 \\
\hline Nombres & 3 & Corazón & 5 & Parce & 3 & Mor & 2 \\
\hline Parce & 5 & Mi amor & 3 & Parcero & 3 & More & 2 \\
\hline Otras & $\ldots$ & Mi vida & 3 & Señor & 3 & Rey & 2 \\
\hline & & Muchis & 3 & Otras & $\ldots$ & Otras & $\ldots$ \\
\hline & & Nena & 3 & & & & \\
\hline & & Parce & 3 & & & & \\
\hline & & Otras & $\ldots$ & & & & \\
\hline Total: $\mathbf{2 7}$ & $\mathbf{4 4}$ & Total: $\mathbf{3 4}$ & $\mathbf{6 7}$ & Total: $\mathbf{3 0}$ & $\mathbf{3 9}$ & Total: $\mathbf{2 2}$ & $\mathbf{3 9}$ \\
\hline
\end{tabular}

Tabla 2: FTN afectivo-positivas 
Siguiendo la misma lógica del cuadro anterior, la comunidad discursiva número uno (FE) en cuanto a la categoría de la afectividad positiva, esto es, la que utiliza palabras pragmática y axiológicamente inocuas a la imagen del destinatario ostenta campos semánticos como Amor o afectividad: (H) amor, amorcito, mor, cariño, ternura. (M) Amor, mamor, mamorch, mi amor, mi amorch, mis amores, mor, ternura, cariño, querido (a): Amistad o compañerismo: (H) parce, parcero, amigo. (M) Amiguis, amiguito, migui, parce; Realeza y/o nobleza: (H) caballero, prince, reina. (M) Princesa; Familia: (H) hermano, primo. (M) mija; Elementos reales o abstractos: (H) bombón, corazón, llave, mi vida. (M) Mi cielo, mi vida, tesoro, muñeca, cosota, cielo, corazón; y Edad: (M) baby, niñas, nena, jovencito.

De estos hallazgos, se advierte que las mujeres utilizan más los sustantivos, adjetivos y sintagmas propios del campo amoroso, siendo preponderantes las derivaciones de la palabra amor. Por su parte, en los hombres se nota un mayor uso de la categoría. Objetos reales y abstractos. Existe, además, una diferencia de 23 usanzas entre el género masculino y femenino, siendo superior en este último. Finalmente, la cadena cohesiva de amor es utilizada indistintamente para ambos géneros; la de amistad y familia tiene en su mayoría como destinatario a un hombre, mientras que en realeza y objetos predominan los receptores femeninos.

Análogamente, la comunidad discursiva número dos (FI) alberga redes léxicas pertenecientes a las categorías Amor o afecto: (H) amor, mor, cariño, cariñito, ternura, querida, ternurita, apreciado, mi amorcito. (M) Amor, amorcito, cariño, mi amor, mor, more; Amistad o compañerismo: (H) Amigazo, amigo, amiguita, compañero, pana, (M) Amigo, camarada; Realeza y/o nobleza: (M) Príncipe, rey; Familia: (H) papá, papi. (M) Hijo; Objetos reales o abstractos: (H) cielo, corazón, Honey (miel). (M) Cielo, cora (corazón), mi vida, muñeca; Edad: (H) bebé, mi viejo. (M) Baby, bebé; y Rol social: (H) señor, señorita, socio, doctor, don.

En consecuencia con esta información, tanto los hombres como las mujeres, en la relación de afectividad, utilizan un mayor número de sinónimos relativos pertenecientes a la categoría de Amor o afecto (26.66\% - 35.89\% respectivamente). Asimismo, los varones crean el campo semántico del rol social (señorial, profesión) carente en su género opuesto.

En síntesis, se presenta en el valor afectivo con carácter positivo una mayor cantidad de variaciones en las mujeres de la FE (34) que de la FI (22), encontrándose además una notable diferencia en el uso que se hace de estas en ambas comunidades discursivas. Por lo demás, es notorio el hecho que la expresión parce, y todas sus variaciones, sea tomada por 
algunos encuestados como displicente, y por otros como cariñosa: aparece con el primer valor 9 veces y con el segundo un total de 15.

\subsection{Valor calificativo}

Como su nombre lo indica, se refiere a todas aquellas construcciones gramaticales que tienen la función de apelar, basadas en rasgos físicos o psicológicos del individuo.

\begin{tabular}{|l|c|l|c|l|c|l|c|}
\hline \multicolumn{4}{|c|}{ Facultad de Educación } & \multicolumn{4}{c|}{ Facultad de Ingeniería } \\
\hline \multicolumn{2}{|c|}{ Hombres (H) } & \multicolumn{2}{c|}{ Mujeres (M) } & \multicolumn{3}{c|}{ Hombres (H) } & \multicolumn{2}{c|}{ Mujeres (M) } \\
\hline Hombres & $U^{*} N P$ & Mujeres & $U^{*} N P$ & Hombres & $U^{*} N P$ & Mujeres & $U^{* *} N P$ \\
\hline Bella & 1 & Bonito & 1 & Flaco & 1 & Hermosa & 2 \\
\hline Bonita & 1 & Guapa & 1 & Hermosa & 2 & Linda & 2 \\
\hline Hermosa & 3 & Guapetona & 2 & Linda & 4 & Negro & 1 \\
\hline Preciosa & 2 & Linda & 1 & Monito & 1 & Pequeña & 1 \\
\hline & & Preciosa & 1 & Preciosa & 1 & & \\
\hline & & & & Preciosura & 1 & & \\
\hline Total: $\mathbf{4}$ & 7 & Total: $\mathbf{5}$ & $\mathbf{6}$ & Total: $\mathbf{6}$ & $\mathbf{1 0}$ & Total: $\mathbf{4}$ & $\mathbf{4}$ \\
\hline
\end{tabular}

Tabla 3: FTN calificativas

Dado el número reducido de variantes, se puede decir que: (1) se presenta un mayor número de vocablos y usos en los hombres de Ingeniería y las mujeres de Educación; (2) todas las palabras, a excepción de negro, flaco y pequeña, pertenecen a la categoría semántica "Belleza"; y (3), se percibe principalmente un receptor femenino en este tipo de vocativos.

\subsection{Valor de lejanía entre desconocidos:}

\begin{tabular}{|l|c|l|c|l|c|l|c|}
\hline \multicolumn{5}{|c|}{ Facultad de Educación } & \multicolumn{5}{c|}{ Facultad de Ingeniería } \\
\hline \multicolumn{1}{|c|}{ Hombres } & $U^{*} N P$ & \multicolumn{1}{|c|}{ Mujeres } & $U^{*} N P$ & Hombres & $U^{*} N P$ & Mujeres & $U^{*} N P$ \\
\hline Amigo & 7 & Amiguito & 2 & Amigo & 2 & Amigo & 3 \\
\hline Caballero & 2 & Caballero & 2 & Chica & 2 & Chico & 4 \\
\hline Chico (a) & 2 & Chico (a) & 4 & Compa & 7 & Compañero & 6 \\
\hline Compa & 5 & Compa & 2 & Cucho & 2 & Muchacho & 5 \\
\hline Compañero & 7 & Compañero & 5 & Parce & 6 & Otras & $\ldots$ \\
\hline Parce & 3 & Joven & 3 & Otras & $\ldots$ & & \\
\hline Vacío & 3 & Parce & 2 & & & & \\
\hline Otras & $\ldots$ & Señor & 2 & & & & \\
\hline & & Otras & $\ldots$ & & & & \\
\hline Total: $\mathbf{1 4}$ & $\mathbf{3 7}$ & Total: $\mathbf{1 5}$ & $\mathbf{3 0}$ & Total: $\mathbf{1 5}$ & $\mathbf{3 0}$ & Total: $\mathbf{1 0}$ & $\mathbf{2 4}$ \\
\hline
\end{tabular}

Tabla 4: FT'N con desconocidos 
En este tipo de valor, se hallan algunas anotaciones generales tales como: (1) se observan campos semánticos similares para ambas facultades, destacando conexiones léxicas como compañerismo (36.56\%), amistad (13.22\%), rol social (9.91\%) y edad - género (28.92\%). Nótese que en los mismos no existe mayor compromiso afectivo por parte del locutor, acudiendo a FTN generales asociadas a la situación de estudiantes, al género o, mayoritariamente, al "impersonal" parce o parcero. (2) En ambas facultades predomina la forma nominal compañero o su acortamiento compa. En cuanto a este valor hay menor cantidad de variantes en las mujeres de Ingeniería y en los hombres de Educación. No obstante, pese a la reducida cuantía de palabras, la mayor utilización de los vocablos se produce en la segunda mencionada. Por su parte, existe una similitud tanto en la suma como en el uso entre las mujeres de la comunidad discursiva uno y los hombres de la dos.

\subsection{Valor de lejanía entre conocidos}

\begin{tabular}{|c|c|c|c|c|c|c|c|}
\hline \multicolumn{4}{|c|}{ Facultad de Educación } & \multicolumn{4}{|c|}{ Facultad de Ingeniería } \\
\hline Hombres & $U^{*} N P$ & Mujeres & $U^{*} N P$ & Hombres & $U^{*} N P$ & Mujeres & $U^{*} N P$ \\
\hline Amigo & 3 & Amigo & 2 & Compa & 4 & Chica & 2 \\
\hline Chico (a) & 2 & Caballero & 4 & Nombre & 3 & Compañero & 2 \\
\hline Compa & 3 & Chico (a) & 4 & Parce & 6 & Nombre & 8 \\
\hline Compañero & 3 & Compa & 3 & Otras & $\ldots$ & Otras & $\ldots$ \\
\hline Nombre & 5 & Compañero & 6 & & & & \\
\hline Parce & 3 & $\begin{array}{l}\text { Muchacho } \\
\text { (a) }\end{array}$ & 2 & & & & \\
\hline & & Nombre & 2 & & & & \\
\hline & & Parce & 3 & & & & \\
\hline & & Vacío & 4 & & & & \\
\hline & & Otras & $\ldots$ & & & & \\
\hline Total: & 25 & Total: & 31 & Total: & 23 & Total: & 15 \\
\hline
\end{tabular}

Tabla 5: FTN con conocidos

Como se contempla, en general, se hallan campos semánticos similares para ambas facultades, destacando conexiones léxicas como compañerismo (37.23\%), amistad (7.44\%), rol social (6.38\%), edad-género (19.14\%) y nombre (18.08\%). Nótese que en los mismos no existe mayor compromiso afectivo por parte del locutor; no obstante, se descubre un grado de empatía superior al valor de lejanía con desconocidos. A diferencia de las axiologías anteriores, aquí el nombre adquiere significación, pues, en concordancia con Carricaburo (1997), Alba de Diego y Sánchez Lobato (1980, p. 104) lo denotativo de la nominación es relevante en estas situaciones donde domina la jerarquía o no existe 
solidaridad (asimetría), ya que, el nivel de distancia es un factor concluyente en el uso del apelativo prototípico.

Figura 1: Proporción entre los valores

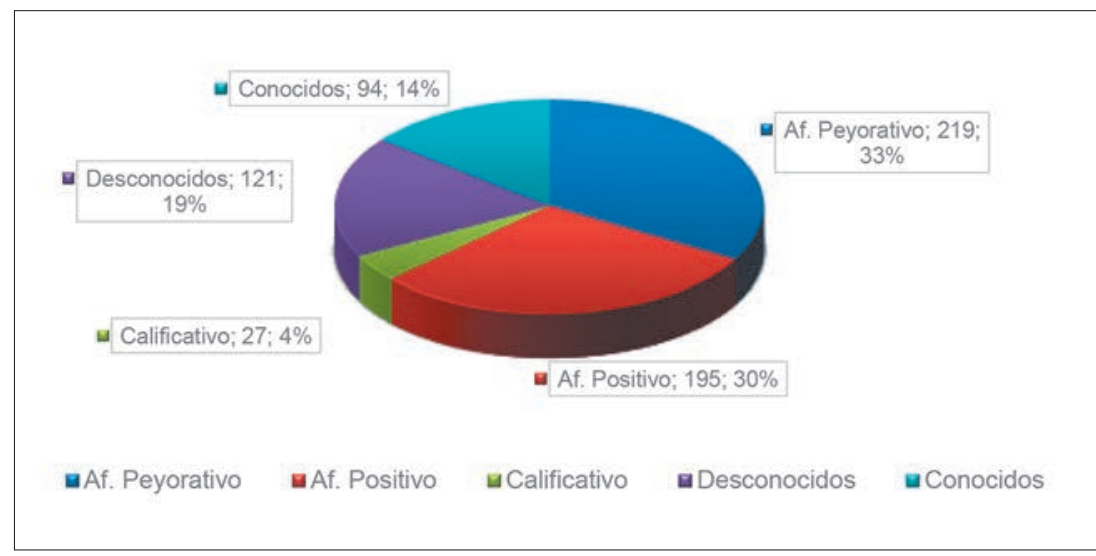

Fuente: elaboración propia

Con el fin de concluir este primer paradigma de análisis, se encuentra que: (1) el valor con mayor cantidad de variantes es el afectivo-peyorativo, los hablantes tienden a escoger palabras de esta categoría antes que de la afectivo-positiva. (2) En contraposición, la muestra deja entrever poca producción de FTN calificativas. (3) Palabras como parcero y su familia léxica es utilizada en casi todos los valores, siendo relacionada por los actantes comunicativos, en su mayoría, como despectivas. (4) La creación de FTN con conocidos o desconocidos no es tan fructífera dado que se acude sobre todo a discernimientos y enunciados explícitos, genéricos o denotativos. (5) Existe mayor creación de FTN en la Facultad de Ingeniería que en la de Educación, lo cual hace pensar que en el campo de estudios humanos se presenta mayor restricción respecto del habla (el buen decir) que en el de lo exacto, serían pertinentes más estudios para respaldar esta hipótesis.

\section{Construcciones lexicales y familias de palabras}

Se comprende como familias léxicas al conjunto de palabras que comparten un mismo lexema o raíz, teniendo en cuenta las variables Facultad y género. Dichas familias se producen, en parte, por ciertas construcciones gramaticales, donde, bien lo plantea Mervyn F. Lang (1990) se observan recursos neologísticos como la sufijación, la combinación y los metaplasmos, estos últimos entendidos como fenómenos lingüísticos en los que se adicionan, suprimen o transponen sílabas o letras en las palabras para la constitución de nuevo léxico o para la comodidad del hablante. Se destacan el paragoge (agrega elemento 
al final) perteneciente al aditamento, y las manifestaciones de acortamiento: aféresis (supresión de un elemento al inicio), síncopa (supresión en el medio) y apócope (supresión al final) en la presente pesquisa. En el ámbito de la supresión (acortamiento), los componentes excluidos no se corresponden con los morfemas establecidos, esto es, la eliminación afecta tanto a la base léxica, como a los morfemas derivativos y a los flexivos.

\subsection{Facultad de Educación}

Son múltiples las variaciones que se producen respecto de las familias léxicas en esta comunidad discursiva. Por una parte, se observa en los hombres la utilización de apócopes en raíces como parcer: parcero $(2 \mathrm{U} * \mathrm{NP})$, parce $\left(14 \mathrm{U}^{*} \mathrm{NP}\right)$; y compañer: compañero (12 $\left.\mathrm{U}^{*} \mathrm{NP}\right)$, compa ( $\left.8 \mathrm{U}^{*} \mathrm{NP}\right)$. Por otra, se hallan acortamientos por aféresis en el lexema am, el cual constituye un paradigma derivativo de donde se desprenden amor ( $\left.7 \mathrm{U}^{*} \mathrm{NP}\right)$, mor (1 $\left.\mathrm{U}^{*} \mathrm{NP}\right)$ y amorcito (U*NP). Se encuentra presente, además, sufijación apreciativa en guev y maric, producida a través del morfema gramatical, derivativo, homogéneo (ón) observado 9 veces en la estructura Güevón y 1 en Maricón. Este se advierte como una variante del morfema gramatical, flexivo, que indica género (a) encontrado 1 vez en el vocablo Güeva y 7 en Marica. El cambio de "Hueva" a "güeva" es un metaplasmo por sustitución de fonema (Antítesis).

Se exteriorizan, asimismo, otras categorías de análisis como los vocablos Gay, (Anglicismo), Hijueputa (Combinación) Nea (Aféresis de la palabra gonorrea) y Prince (Apócope de la palabra princesa), todos con una frecuencia absoluta de 1.

Por otra parte, las mujeres encuestadas en la FE poseen en su inventario léxico familias como: (1) am : mi amor (3 $\left.U^{*} N P\right)$, mor (1 $\left.U^{*} N P\right)$, mi amorch $\left(1 U^{*} N P\right)$, amor ( $\left.6 U^{*} N P\right)$, mamorch $\left(1 U^{*} N P\right)$; donde, se produce una aféresis en mor; se localiza el sustantivo amor sin ningún tipo de alteración; se presenta un sintagma nominal combinado, miamor, en el que se observa la unión del pronombre posesivo y el sustantivo a partir de un proceso de diptongación; se crea una modificación por combinación y paragoge en mamorch, en la que se cambia el sonido vibrante simple /r/por el africado palatal/č/, un fonema asociado generalmente con lo afectivo, representación fónica que tiene incidencia morfológica en la estructuración de la palabra.

(2) grill: grilla $\left(1 U^{*} N P\right)$, grillis $\left(2 U^{*} N P\right)$; maric: marica $\left(1 U^{*} N P\right)$, mariconcita (1 $\left.U^{*} N P\right)$; chic: chica $\left(8 U^{*} N P\right)$, chicola $\left(1 U^{*} N P\right)$; en las que pueden evidenciarse como Fórmulas de Tratamiento Nominal, sustantivos que a la vez presentan un fenómeno de sufijación apreciativa, puesto que morfemas gramaticales, derivativos con una función aumentativa o diminutiva como (on), (cit), (is), se introducen en el lexema. Así mismo, se halla una variante alomórfica en el afijo (uel-a) ya que su realización se da a través del 
morfo (ola) en chicola. Debe resaltarse también que, varias de las construcciones que se presentan en las familias léxicas son agramaticales y, por tanto, se generan a partir del uso que cada hablante hace de la lengua. Así, morfemas como (is) -en la palabra grillis- y (ola) -en la palabra chicola- son inexistentes para la norma prescriptiva vigente.

(3) compañer: compa (6 $\left.U^{*} N P\right)$, compañero (12 $\left.U^{*} N P\right)$. Aquí se contempla un fenómeno de apócope, en donde el vocablo compañero pierde las dos últimas sílabas (ñero), presentándose una disminución que afecta al morfema base.

(4) señor: señor $\left(3 U^{*} N P\right)$, señora $\left(1 U^{*} N P\right)$, señorita $\left(1 U^{*} N P\right)$. La variante lexema, constituye una raíz libre, utilizada para el género masculino, que pasa a ser empleada para el femenino al agregársele el morfema gramatical flexivo que indica género (a). Para el caso de la variante señorita se hace uso de la sufijación apreciativa (it), más el morfema gramatical flexivo de género.

Vocablos como Grillis (Construcción agramatical que altera el lexema introduciéndose como un sufijo de afectividad / $1 \mathrm{U} * \mathrm{NP}$ ), Guapetona (Sustantivo con sufijación apreciativa / 2 usos), Güevón (Metaplasmo- Antítesis / 4 U*NP ), Mi Vida (Sintagma Nominal), Muchis (Sufijación Apreciativa / 3 U*NP), Parce (Apócope), Sexy (Anglicismo), acortamiento del nombre $\left(1 \mathrm{U}^{*} \mathrm{NP}\right)$, nombres $\left(3 \mathrm{U}^{*} \mathrm{NP}\right)$, entre otras (cosota, maldita bitch, maldita buena, migui, nea, malparida...) constituyen palabras que no teniendo familias léxicas, albergan fenómenos lingüísticos particulares.

Ahora, y contrastando ambas poblaciones, se encuentra que los varones tienden a utilizar FTN analizables desde los fenómenos de acortamiento, ya sea por apócope o por aféresis, y sufijaciones apreciativas a través de morfemas aumentativos y en muy pocos casos diminutivos. Presentan ausencia de familias léxicas de carácter afectivo como las que pertenecen a los lexemas grill, chic, señ respecto de las halladas en las féminas de la muestra. Mientras tanto, estas se inclinan hacia la utilización de FTN examinables desde los fenómenos de sufijación apreciativa con morfemas principalmente diminutivos, sintagmas nominales y acortamientos por apócope. Además, presentan ausencia de familias léxicas peyorativas de las raíces güev y parcer respecto de las halladas en los hombres encuestados.

En cuanto a las palabras que no pertenecen a las familias léxicas específicas, pero que son utilizadas como FTN, puede decirse que en las mujeres se manifiestan como adjetivos sustantivados que expresan afectividad, o cualidades particulares. Se registra un menor uso de acortamientos del nombre y de la utilización como tal de este. En los hombres, por su parte, se vislumbra un mayor uso de palabras peyorativas y de sufijos aumentativos en los vocablos que designan cierta cualidad, así como una mayor utilización de apodos respecto de las mujeres y un menor uso de los acortamientos del nombre. 


\subsection{Facultad de Ingeniería:}

Esta comunidad discursiva, en donde prima una mayor cantidad de familias léxicas, tiene como elemento diferencial el predominio de palabras compuestas y la superior producción de metaplasmos entre los hablantes.

Inicialmente, siguiendo el mismo orden utilizado en la comunidad uno, es menester decir que la población masculina de la muestra posee palabras derivadas con rasgos análogos a la FE en raíces como güev: güeva (1 U*NP), güevón (13 U*NP); maric: marica (13 U*NP), maricón ( $\left.4 \mathrm{U}^{*} \mathrm{NP}\right)$; y compañer: compa (13 $\left.\mathrm{U}^{*} \mathrm{NP}\right)$, compañero (3 $\left.\mathrm{U}^{*} \mathrm{NP}\right)$. A la vez, se encuentran en el inventario lexical de los emisores los semantemas:

(1) parcer: parce $(26 \mathrm{U} * \mathrm{NP})$, parcero $(9 \mathrm{U} * \mathrm{NP})$, parcerito $(1 \mathrm{U} * \mathrm{NP})$. Donde se presenta, en primera instancia, un acortamiento por apócope en la palabra parce; $\mathrm{y}$, en una segunda, se manifiesta una sufijación apreciativa de diminutivo en la palabra parcerito.

(2) amigu: amigo (6 $\left.\mathrm{U}^{*} \mathrm{NP}\right)$, amigazo $\left(1 \mathrm{U}^{*} \mathrm{NP}\right)$, amiguita $\left(1 \mathrm{U}^{*} \mathrm{NP}\right)$; cariñ cariño $\left(2 \mathrm{U}^{*} \mathrm{NP}\right)$, cariñito $\left(1 \mathrm{U}^{*} \mathrm{NP}\right)$. En las que existe sufijación apreciativa respecto de las palabras primitivas amigo y cariño, debido a que los posfijos (azo) e (it) expresan cercanía, emotividad o afectividad a través de sus funciones aumentativas y diminutivas respectivamente.

(3) am: amor $\left(5 \mathrm{U}^{*} \mathrm{NP}\right)$, mi amorcito $\left(1 \mathrm{U}^{*} \mathrm{NP}\right)$, mor $\left(1 \mathrm{U}^{*} \mathrm{NP}\right)$; pap: papá (1 $\left.\mathrm{U}^{*} \mathrm{NP}\right)$, papi (1 $\left.\mathrm{U}^{*} \mathrm{NP}\right)$. Ambas categorías exteriorizan un fenómeno de acortamiento. En la primera se presenta el tipo aféresis ya que se omite el inicio de la palabra amor (a), mientras que en la segunda se da una apócope en la que se suprime la sílaba final (to) del diminutivo. Por otra parte, la familia inicial presenta también un sintagma nominal (mi amorcito) compuesto por un pronombre posesivo y un sustantivo con la presencia de un sufijo apreciativo diminutivo (cit).

(4) señor: señor ( $\left.5 \mathrm{U}^{*} \mathrm{NP}\right)$, señorita ( $\left.4 \mathrm{U}^{*} \mathrm{NP}\right)$. En esta puede notarse, debido a que la raíz es libre, dos variantes que aluden a una diferenciación de género. Así, el lexema señor se utiliza para referenciar a los hombres y para el caso de referirse a las mujeres se utiliza una sufijación apreciativa (it) más el morfema gramatical, flexivo que indica género (a).

(5) perr: perra (2), perris (1), perro (2). Los fenómenos que se pueden evidenciar dentro de este grupo de variantes son dos: el primero apunta hacia una distinción de género con los morfemas flexivos $(0$ - a), y el segundo se refiere a una construcción agramatical 
en la palabra perris, donde la partícula (is) altera al semantema introduciéndose como un postfijo que expresa afectividad.

Vocablos como Pelao ( 4 U*NP / síncopa), Abortomula (1 U*NP / combinación), Brutus (1 U*NP / sonido "latinizado"), Cabrón (1 U*NP / aumentativo-peyorativo), Carechimba (1 U*NP / combinación), Careñame (1 U*NP / combinación), Chimbón (1 U*NP / aumentativo o cualidad), Chupavergas ( 1 U*NP / combinación), Cochobis (1 U*NP / reestructuración silábica de bizcocho), Gay (1 U*NP / anglicismo), Hijueputa (5 U*NP / combinación), Malparido ( $4 \mathrm{U} * \mathrm{NP} /$ combinación), Marranomono (2 U*NP / combinación), Marranopadrón (1 U*NP / combinación), Mi viejo (1 U*NP / sintagma nominal), Nea (2 U*NP / aféresis, parlache), Ñero (1 U*NP / aféresis), y Ñómpiro (1 U*NP/ parlache), Soplamondá (1 U*NP / combinación), Travis (1 U*NP / morfema is- apreciativo) son palabras que no constituyendo familias lexicales poseen fenómenos lingüísticos específicos.

Entre tanto, poniendo la mirada sobre la población femenina, se observan familias léxicas pertenecientes a las raíces: (1) guev: güeva (1 U*NP), güevón (2 U*NP). FTN que constituye una constante en el estudio de las muestras, véase el análisis realizado a los hombres de la Facultad de Educación; (2) perr: perra ( $\left.1 \mathrm{U}^{*} \mathrm{NP}\right)$, perris (1 U*NP). En este grupo léxico se distingue para el caso del sustantivo perra, una variante de género con el morfema (a). Para perris se evidencia nuevamente la partícula apreciativa (is); (3) amig: amigo (6 U*NP), amiguito (1 U*NP). Se da una manifestación de sufijación respecto del sustantivo masculino amigo con el diminutivo (it). (4) am: amor (9 $\left.\mathrm{U}^{*} \mathrm{NP}\right)$, amorcito (1 $\left.\mathrm{U}^{*} \mathrm{NP}\right)$, mor $(4 \mathrm{U} * \mathrm{NP})$, more $(2 \mathrm{U} * \mathrm{NP})$, mi amor $(1 \mathrm{U} * \mathrm{NP})$. En esta familia gramatical se exteriorizan varios fenómenos; se advierte la utilización del sustantivo amor (morfema libre), del sintagma nominal mi amor, compuesto por un pronombre posesivo más un sustantivo y el uso de un diminutivo en amorcito. Se da, de igual manera, un acortamiento tipo aféresis en mor. Finalmente hace presencia un fenómeno para el caso de la variante more, en la que se observa la utilización de una palabra extranjera del italiano (amore) que a su vez sufre un acortamiento tipo aféresis. (5) compañer: compa (2 U*NP), compañerito ( $\left.1 \mathrm{U}^{*} \mathrm{NP}\right)$, compañero $\left(8 \mathrm{U}^{*} \mathrm{NP}\right)$. Donde se halla una apócope en la palabra compa, y sufijación apreciativa en la palabra compañerito.

Cualificando otros vocablos sin grupos de semantemas se encuentran Baby (1 U*NP / anglicismo), Cora (1 U*NP / apócope), Gransosioso (1 U*NP / combinación), Nea (1 U*NP / aféresis, parlache), Osito (1 U*NP / diminutivo), Parce (8 U*NP / apócope), Acortamientos del nombre ( $\left.4 \mathrm{U}^{*} \mathrm{NP}\right)$ y Apodos $\left(5 \mathrm{U}^{*} \mathrm{NP}\right)$ como elementos relevantes. 
Finalmente, al realizar los contrastes respectivos, se acierta al decir que entre los hombres de la comunidad discursiva número dos se evidencia una inclinación por el uso de palabras que sufren acortamientos de tipo aféresis y apócope particularmente. También es preponderante el empleo de partículas apreciativas en los sustantivos utilizados como FTN, donde sobresalen los diminutivos. En cuanto a las palabras que no poseen familia léxica, hay un dominio del masculino en la utilización de las mismas que señalan un trato peyorativo. Respecto de la mujer, los términos empleados, en su mayoría, son adjetivos que expresan afectividad y cualidades positivas. De igual manera, en la población encuestada el uso de palabras combinadas como FTN es significativo. Por su parte, las mujeres emplean términos que sufren acortamientos en menor medida que los varones, observándose mayor usanza de elementos que expresan afectividad en los sustantivos. En lo que respecta a las palabras no poseedoras de familias léxicas, se mantiene el uso de términos despreciativos para el género masculino, y para el femenino aquellos que están en el plano de lo afectivo positivo.

Así también, dentro del análisis realizado a cada facultad, puede decirse que en la comunidad de Ingeniería se utiliza mayor número de combinaciones con términos que no son propios de Medellín (variable diatópica) y que, en su mayoría, son más cercanos y/o propios a la región del Caribe. En cuanto a las familias léxicas, se tiene que estas son comunes en ambas dependencias académicas. Con respecto a la población, en las mujeres encuestadas de ambas comunidades hay una tendencia por la utilización de sufijos apreciativos con morfemas diminutivos mayor que en los hombres. Por su parte, la población masculina de la FI presenta, en relación con la población femenina de su misma comunidad y de la de educación, mayor número de familias léxicas, mayor número de palabras combinadas y mayor uso de palabras despectivas.

\section{Conclusiones}

A través del presente artículo se evidencian diversas formas en las que los hablantes de dos comunidades académicas específicas utilizan FTN para referirse a sus iguales. En este sentido, los grupos derivativos analizados en el total de los encuestados se caracterizan por la presencia de acortamientos, ya sea por apócope o aféresis; la utilización de sufijos apreciativos con morfemas aumentativos, por lo general en los hombres; morfemas diminutivos, utilizados con mayor frecuencia por las mujeres y por la construcción de sintagmas nominales. Se nota, en cuanto a palabras propias del parlache, que es necesario actualizar y estudiar la inmersión de algunos morfemas a los textos académicos, como sucede con el ya mencionado (is) pues, en lo que refiere a estos, no existen suficientes estudios desde perspectivas morfosintácticas. 
Por otro lado, las FTN que no se constituyen como familias léxicas se catalogan en apodos, acortamientos del nombre, el nombre mismo, extranjerismos y palabras combinadas. Se tiene que, en la población masculina no hay uso del nombre propio y/o acortamiento del mismo, mientras que en la población femenina hay ausencia del nombre propio y de los apodos. Cabe mencionar que en las FTN, en lo que compete al género de los hablantes, no se observan aspectos significativos a la hora de escoger un apelativo sobre otro, siendo frecuentes aquellos que no acarrean consigo un morfema flexivo que indique género como parce, marica, etcétera.

Finalmente, en cuanto a lo pragmasemántico, es necesario decir que el espacio de cercanía propio de una relación entre amigos, novios y demás, está constituido por una multiplicidad de FTN que son resignificadas y recontextualizadas desde otras situaciones comunicativas al campo mencionado, así, palabras del paradigma despectivo adquieren un matiz positivo, y enunciados que usualmente son del tipo calificativo transmutan al campo de la función apelativa. En estos fenómenos, se vuelve irrelevante la veracidad y correspondencia de dichas fórmulas con la realidad empírica de los individuos, pues, la notabilidad comunicativa radica en los entramados significacionales instaurados entre aquellas personas. De esta manera, las FTN adquieren tonalidades diversas para situaciones expresivas disímiles, donde, tanto las derivaciones morfológicas como sus instancias semánticas son reelaboradas, matizadas y transmutadas de acuerdo con intenciones digresivas, afectivas o de formalidad.

\section{Referencias bibliográficas}

Almela Pérez, R. (1999). Procedimientos de formación de palabras en español. Barcelona: Ariel. Arcelus Ulibarrena, J. M. (1980). Fórmulas de tratamiento en el español actual. Actas del Congreso de AEPE. Budapest: Academia de las Ciencias de Hungría.

Berruto, G. (2003). Fondamenti di sociolinguistica. Roma-Bari: Laterza

Carricaburo, N. (1997). Las fórmulas de tratamiento en el español actual. Madrid: ARCOS/ LIBROS SL.

Casado Velarde, M. (1999). Otros procedimientos morfológicos: Acortamientos, formación de siglas y acrónimos. En: Bosque y Demonte. (Dirs). Gramática descriptiva de la lengua española (Tomo III). Madrid: Espasa, pp. 5075-5096. 
Castellano, M. (2008). Fórmulas de tratamiento nominales para la pareja en el habla juvenil medellinense. Íkala, Revista de Lenguaje y Cultura. 13(20), 163-181.

Castellano, M. (2011). Caracterización morfológica de las fórmulas de tratamiento nominales en el habla de Medellín. Revista Virtual Universidad Católica del Norte, (32), 220-246.

Castellano, M. (2012). Cortesía verbal y fórmulas de tratamiento nominales: Análisis pragmático de las fórmulas de tratamiento nominales en el habla de Medellín. Cuadernos de Lingüística Hispánica, (20), 41 - 56.

Dumitrscu, D. (1975). Notas comparativas sobre el tratamiento en español y rumano. Bulletin de la Societé Roumanie de Linguistique Romane, (9), 81-86.

Flores, L. (1954). Algunas fórmulas de tratamiento en el español del departamento de Antioquia. Bogotá: Instituto Caro y Cuervo.

Fontanella, B. (1999). Sistemas pronominales de tratamiento usados en el mundo hispánico. En: Bosque y Demonte (Dirs). Gramática descriptiva de la lengua española (Tomo I). Madrid: Espasa, pp. 1399-1426.

Lang, M. (1990). Formación de palabras en español. Madrid: Cátedra.

Lázaro Mora, F. (1999). La derivación apreciativa. En: Bosque y Demonte (Dirs). Gramática descriptiva de la lengua española (Tomo III). Madrid: Espasa, pp. 4645-4682.

Lobato, J. S., \& de Diego, V. A. (1980). Tratamiento y juventud en la lengua hablada: Aspectos sociolingüísticos. Boletín de la Real Academia Española, 60(219), 95-130.

Molina, I. (2002). Evolución de las fórmulas de tratamiento en la juventud madrileña a lo largo del siglo XX: un estudio en tiempo real. En Rodríguez, F. (Coord.). El lenguaje de los jóvenes. Barcelona: Ariel social, pp. 97-135.

Moreno, F. (2009). Principios de sociolingüística y sociología del lenguaje [4ª ed.]. Barcelona: Ariel Lingüística

Pedroviejo, J. (2006). Un estudio sociolingüístico. Sistemas de tratamiento de la juventud de Valladolid. Valladolid: Revista electrónica de estudios filológicos.

Trudgill, P. (1975). Sociolinguistics and Scots Dialects. En J.D. McClure (ed). The Scots Language in Education: Association for Scottish Literary Studies. Occasional Papers No. 3. Aberdeen: Association for Scottish Literary Studies. pp. 28-34. 